Aim of the study: Primary bone tumours are relatively rare, but their diagnosis and treatment is difficult and connected with a high risk of complications. The goal of this report is a retrospective evaluation of outcomes in patients with primary tumours of the humerus treated in our centre with the use of modular endoprosthetic reconstruction.

Material and methods: Currently, surgical treatment is a standard procedure for local therapy, with wide tumour-free margin resection after a planned multidisciplinary and individualised strategy of tumour management based on the diagnostic biopsy result. The best option for patients to avoid disability is simultaneous surgical reconstruction aiming to spare the limb and its functionality. Results: In this report, we present the results of treatment of our 11 adult patients suffering from primary bone tumours of the humerus, who have undergone wide bone resection followed by reconstruction with the use of a modular MUTARS $®$ endoprosthesis.

Conclusions: The study showed that prosthetic reconstruction of the resected humerus due to a primary bone tumour is safe and acceptable for patients; despite the fact that limitation of active abduction of the shoulder is up to 20 grades, this surgical procedure provides satisfactory limb function.

Key words: primary bone tumours, humerus bone resection, shoulder joint reconstruction, modular tumour endoprosthesis.

Contemp Oncol (Pozn) 2017; 21 (3): 228-231 DOI: https://doi.org/10.5114/wo.2017.70113

\section{Treatment results of adult patients with primary bone tumours of the humerus with the use of the oncological modular endoprosthesis}

\author{
Tomasz Goryń, Andrzej Pieńkowski, Andrzej Komor, Wirginiusz Dziewirski, \\ Marcin Zdzienicki, Maria Koziot, Piotr Rutkowski
}

Department of Soft Tissue/Bone Sarcoma and Melanoma, Memorial Cancer Center and Institute of Oncology, Warsaw, Poland

\section{Introduction}

Primary bone malignant tumours (namely sarcoma) occur rarely but tend to be both a diagnostic and therapeutic challenge. They constitute about $1 \%$ of all bone lesions and less than $1 \%$ of all malignant tumours in adults. The most common sites of occurrence are long bones, especially the distal femur, proximal tibia, and proximal humerus. The most common histopathological diagnoses are osteosarcoma, chondrosarcoma, and giant cell tumour of bone (GCTB). The best treatment results can be achieved with the use of multimodal and individualised therapy in highly specialised centres. Currently, wide surgical resection with tumour-free margins is the best means of local treatment, followed by reconstruction of the resected bone with endoprosthesis or allograft if possible. This kind of treatment can be used in about $80-90 \%$ of patients, giving them the best option to achieve good functional effect and reducing the risk of disability. However, in some cases the only possible surgical treatment is amputation or resection without reconstruction. In most cases the surgical procedure is combined with perioperative chemotherapy (especially in osteosarcoma and Ewing sarcoma) [1]. In our centre, in locally advanced GCTB, we also use neoadjuvant therapy with denosumab [2]. The patient is given 7-10 cycles of denosumab prior to the operation. Our goal is to achieve satisfactory tumour calcification, which facilitates tumour resection and allows better local control to achieve microscopically radical resection. Endoprosthetic reconstruction is a procedure that bears a high risk of possible complications such as prolonged healing time, higher risk of infection, and in some cases the need for re-operation. The use of an endoprosthesis with previous wide muscle/ rotator cuff resection could cause proximal subluxation, instability, and a reduction in functional range of motion. Due to complex shoulder anatomy, such a procedure presents a challenge for the surgeon and is connected with a risk of long-term consequences.

\section{Material and methods}

In this retrospective analysis, we included 11 patients treated in our centre between 2011 and 2015. Since 2011 we have been using modular reconstructive endoprostheses MUTARS ${ }^{\circledR}$ (Modular Universal Tumour And Revision System; www.implantcast.de) after resection of humerus bone tumours if such reconstruction is feasible. All patients were evaluated by a multidisciplinary team in our centre at the initial diagnosis. There were 5 women and 6 men aged between 23 and 78 years, average age 39 years. Ten patients were treated due to a primary bone tumour located in the proximal part of the humerus, and one patient was treated due to a chondrosarco- 
ma comprising of the whole humerus. Five patients were treated for osteosarcoma, 2 for chondrosarcoma, and 4 for GCTB (Campanacci stage 3). In 7 patients the diagnosis was based on a biopsy of the tumour performed in our department, whereas in 4 patients the preoperative biopsy was performed in another hospital. Patients with diagnosis of osteosarcoma received perioperative chemotherapy based on the doxorubicin and cisplatin regimen up to nine courses -3 preoperative and up to 6 postoperative. Patients with GCTB received neoadjuvant denosumab $X$-geva ${ }^{\circledR}$. The number of courses and time of treatment were different in each patient and varied from 9 to 44 courses (7-43 months) before operation. The date of the operation was determined by the stage of tumour calcification as demonstrated on computed tomography (CT) and X-ray. Patients diagnosed with chondrosarcoma were treated primarily by surgical resection. In all patients with osteosarcoma we routinely performed positron emission tomography (PET-CT) and chest CT for the assessment of the presence of metastatic disease. In all patients qualified for surgical treatment no metastatic progression was detected. Primary tumour extent was assessed with magnetic resonance imaging (MRI) and X-ray. In all patients the disease was advanced only locally: stage $\mathrm{Ila}$ and $\mathrm{Ilb}$ according to TNM AJCC classification [3]. The description of analysed patients is shown in Table 1 .

The surgical procedures were performed by two surgeons (TG, AP) experienced in musculoskeletal surgery. We used an anterior transdeltoid approach with resection of the biopsy scar en bloc with the specimen. Then we dissected the tumour and distal tumour-free part of the humerus with a cuff of normal tissue. The humerus was cut at least $2.0 \mathrm{~cm}$ distally from the distal part of the malignant lesion. The bone marrow from the distal part of the humerus was sent for intraoperative frozen section to confirm a negative margin. Then the MUTARS ${ }^{\circledR}$ endoprosthesis system was implanted typically. In one patient, we resected the whole humerus with an additional endoprosthetic reconstruction of the elbow joint (Fig. 1, 2). If possible we tried to reattach the rotator cuff tendons to the holes on the collar of the endoprosthesis and other muscles to the Trevira mesh tube attached to the endoprosthesis. The mean length of the humeral excision was $15.5 \mathrm{~cm}$ (ranging from 8.5 to $36 \mathrm{~cm}$ ). All patients received prophylactic antibiotics in the perioperative period according to standard procedures in our centre (1.0 g cefazolin) before skin incision and up to 6 days after surgery $-2.0 \mathrm{~g}$ per day. The rehabilitation started on the second day after operation. Patients were discharged from the ward 5-7 days after surgery and are continuing physical therapy.

During follow-up, patients underwent a clinical examination, and an X-ray of the humerus was performed every 3 months for the first year and then every 6 months, to exclude local recurrence. In patients treated due to osteosarcoma or Ewing sarcoma we routinely performed lung and abdomen CT scans every 6 months to exclude metastatic disease. The mean follow-up period was 31 months (range 8-65 months).

\section{Results}

$\mathrm{RO}$ resections were performed microscopically in all patients. In 10 patients type I intra-articular proximal humeral resection (according to Malawer et al.) [4] with arthroplasty was performed, and in one patient total humeral resection with total arthroplasty was performed. None of the treated patients developed local recurrence. In one patient treated for osteosarcoma, metastatic disease was detected six months after surgery. He underwent lung metastasectomy thereafter. Other patients treated due to chondrosarcoma developed regional lymph node metastases.

In terms of complications, superficial infection occurred in five patients (45\%), which required prolonged local conservative treatment. Four patients developed a deep tissue infection and one of them had to be re-operated. In that patient, we had to remove the endoprosthesis due to severe infection. On the $\mathrm{X}$-ray examination there were no signs of prosthetic loosening in any patient. Functional results were satisfactory for all patients (Fig. 3). None of the patients, except one, were able to abduct their shoulder more than $20^{\circ}$. The mean score using the Musculoskeletal Tumour Society (MSTS) proximal arm/shoulder/scapula scoring system - International Symposium on Limb Sal-

Table 1. The detailed characteristics of the patients

\begin{tabular}{|c|c|c|c|c|c|}
\hline No. & Gender & Age & Histopathology & G & Concomitant diseases \\
\hline 1 & Female & 55 & Osteosarcoma & G1 & \\
\hline 2 & Male & 38 & Osteosarcoma & G3 & $\mathrm{HCV}$ \\
\hline 3 & Female & 27 & Osteosarcoma & G3 & \\
\hline 4 & Female & 38 & Osteosarcoma & G3 & \\
\hline 5 & Female & 48 & Osteosarcoma & G3 & \\
\hline 6 & Male & 37 & Chondrosarcoma & G3 & \\
\hline 7 & Male & 78 & Chondrosarcoma & G2 & Prostate cancer \\
\hline 8 & Male & 30 & GCTB & & \\
\hline 9 & Male & 29 & GCTB & & \\
\hline 10 & Male & 26 & GCTB & & \\
\hline 11 & Female & 23 & GCTB & & \\
\hline
\end{tabular}



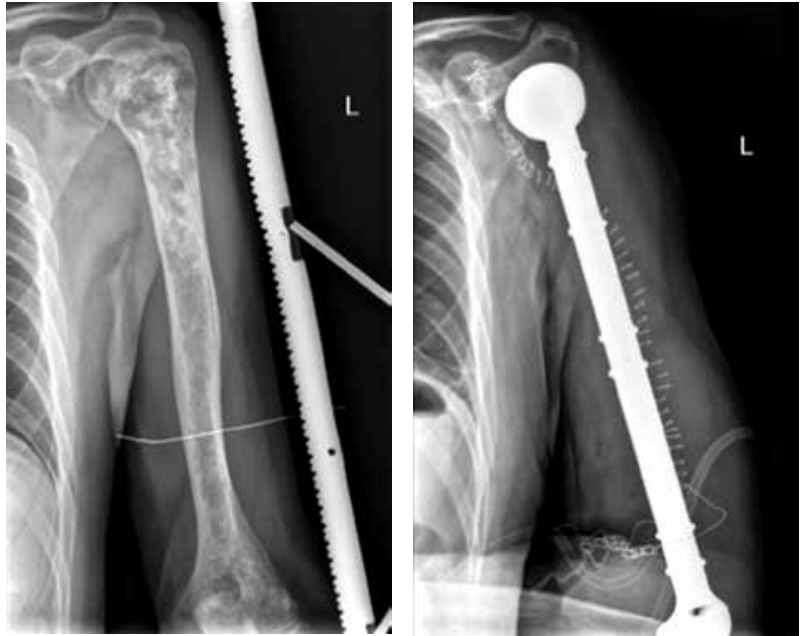

Fig. 1A, B. Patients with chondrosarcoma G2 of left humerus before and after operation (plain X-ray)

vage was $74 \%$. Patients with proximal humerus replacement showed an MSTS score of 15 points - 42\% (10-29 points; 34-83\%). Cosmetic results were good. During follow-up no local recurrence was observed.

\section{Discussion}

The optimal treatment methods in patients with a primary bone tumour located in the proximal humerus tend to be enblock Tickhoff-Linberg resection and various types of reconstruction to stabilise the joint, making stable the skeletal construct and preserving some range of movement in the painful limb. In this study, we analysed a homogenous series of 11 adult patients treated due to
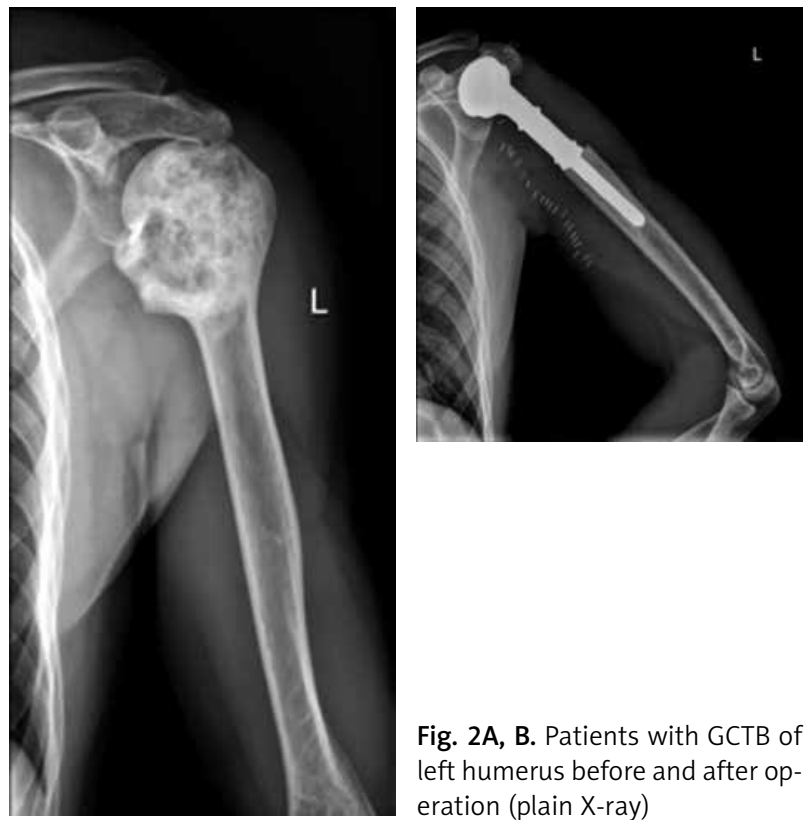

Fig. 2A, B. Patients with GCTB of left humerus before and after operation (plain X-ray)

primary bone sarcomas of the humerus with endoprosthetic reconstruction. We confirmed the data from other studies that this kind of reconstruction is safe for patients and it has comparable functional results in relation to other reconstructive techniques; moreover, we have provided these data using for reconstructions only oncological modular prosthesis, and we limited the patients to primary bone tumours of the humerus [5-8]. In our study, we have been able to show that prosthetic reconstruction gives satisfactory functional effect for patients. The crucial point for prosthetic implantation is adequate soft tissue
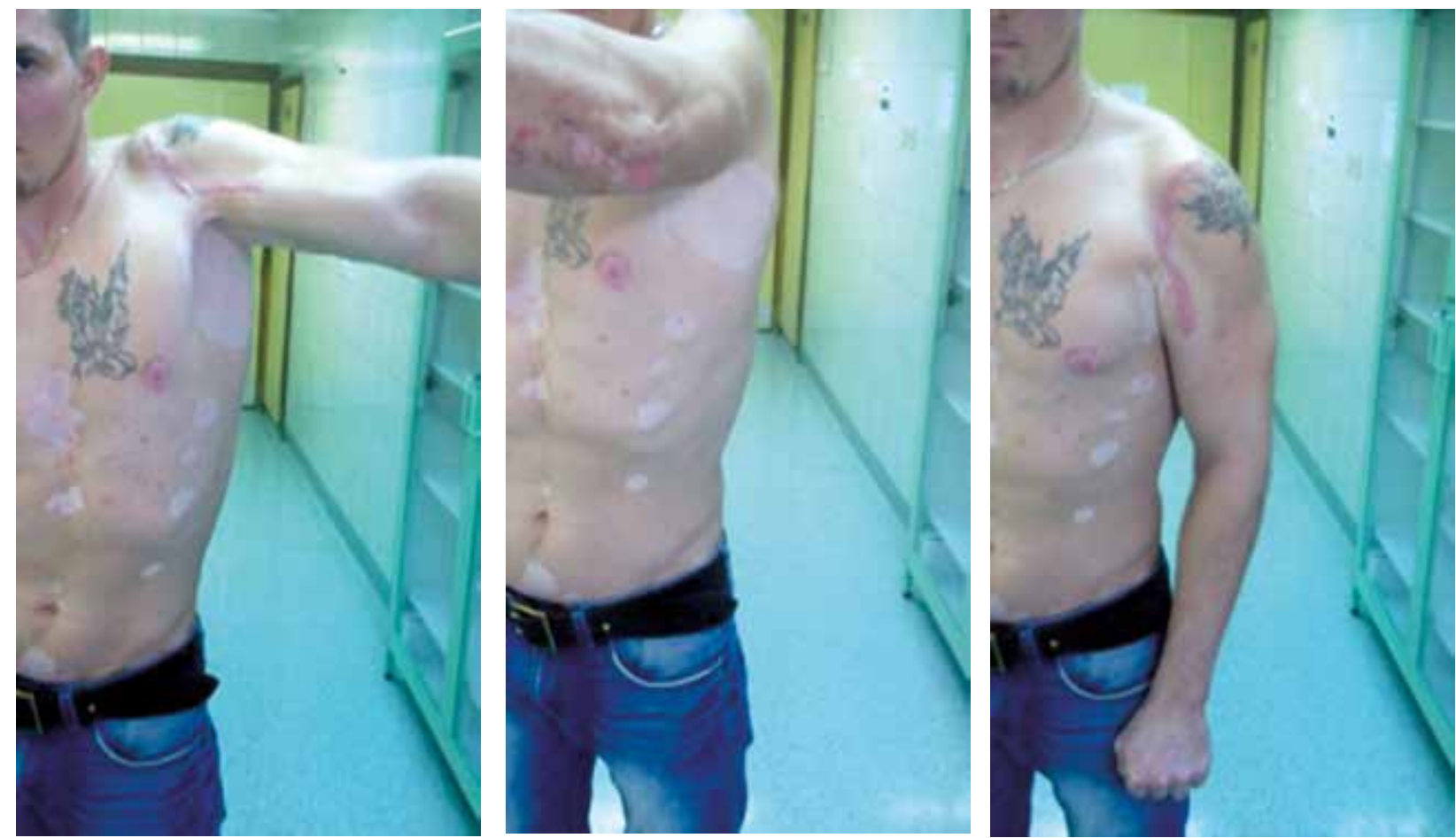

Fig. 3A-C. Functional result of flexion and abduction in patient after operation of GCTB resection and reconstruction with modular prosthesis 
coverage and reconstruction. Although shoulder reconstructions provide stability, active range of movement is sacrificed as is indicated by very limited active abduction. The use of a synthetic mesh tube can improve shoulder function $[9,10]$, and we have used this device in all cases. The low MSTS score after proximal humerus replacement is the result of markedly impaired function of the shoulder joint. Achieving very good functional results of reconstruction of shoulder joint function is not possible because of the resection of the rotator cuff muscles and division of the subscapularis, deltoid, pectoralis major muscles, etc. These endoprostheses have predominantly a spacer function when function in the elbow and wrist is preserved. Our results are similar to those of Shin et al. [7], Tunn et al. [11], and Fabroni et al. [12]. It is also important that the endoprosthetic reconstructions are easy to perform by experienced surgeons and allow the patient to start immediate rehabilitation, which is important also from a psychological point of view. Nevertheless local, non-sarcoma-related complications are relatively frequent $[5,11]$. A relatively short period of observation remains the main limitation of our study, but we keep these patients in further active follow-up.

In conclusion, the study showed that prosthetic reconstruction of the resected humerus due to a primary bone tumour is safe and acceptable for patients; despite the fact that limitation of active abduction of the shoulder is up to 20 grades, this surgical procedure provides satisfactory limb function.

The authors declare no conflict of interest.

\section{References}

1. ESMO/European Sarcoma Network Working Group. Bone sarcomas: ESMO Clinical Practice Guidelines for diagnosis, treatment and follow-up. Ann Oncol 2014; 25 Suppl 3: iii113-23. Erratum in: Ann Oncol 2015; 26 Suppl 5: v174-7.

2. Rutkowski P, Ferrari S, Grimer RJ, et al. Surgical Downstaging in an Open-Label Phase II Trial of Denosumab in Patients with Giant Cell Tumor of Bone. Ann Surg Oncol 2015; 22: 2860-8.

3. Edge S, et al. AJCC Cancer Staging Manual, Springer-Verlag, New York, 2010.

4. Malawer MM, Meller I, Dunham WK. A new surgical classification system for shoulder-girdle resections. Analysis of 38 patients. Clin Orthop Relat Res 1991; 267: 33-44.

5. Kitagawa Y, Thai DM, Choong PF. Reconstructions of the shoulder following tumour resection. J Orthop Surg (Hong Kong) 2007; 15: 201-6.

6. Liu T, Zhang Q, Guo X, Zhang X, Li Z, Li X. Treatment and outcome of malignant bone tumors of the proximal humerus: biological versus endoprosthetic reconstruction. BMC Musculoskelet Disord 2014; 15: 69 .

7. Shin $\mathrm{KH}$, Park $\mathrm{HJ}$, Yoo JH, Hahn SB. Reconstructive surgery in primary malignant and aggressive benign bone tumour of the proximal humerus. Yonsei Med J 2000; 41: 304-11.

8. Puri A, Gulia A. The results of total humeral replacement following excision for primary bone tumour. J Bone Joint Surg Br 2012; 94: 1277-81.

9. Ferguson PC. CORR Insights $\left({ }^{\circledR}\right)$ : synthetic mesh improves shoulder function after intraarticular resection and prosthetic replacement of proximal humerus. Clin Orthop Relat Res 2015; 473: 1735-6.
10. Tang X, Guo W, Yang R, Tang S, Ji T. Synthetic mesh improves shoulder function after intraarticular resection and prosthetic replacement of proximal humerus. Clin Orthop Relat Res 2015; 473: 1464-71.

11. Tunn PU, Pomraenke D, Goerling U, Hohenberger P. Functional outcome after endoprosthetic limb-salvage therapy of primary bone tumours - a comparative analysis using the MSTS score, the TESS and the RNL index. Int Orthop 2008; 32: 619-25.

12. Fabroni RH, Castagno A, Aguilera AL, Steverlynck AM, Zeballos J. Long term results of limb salvage with the Fabroni custom made endoprosthesis. Clin Orthop 1999; 358: 41-52.

13. Shi SF, Dong Y, Zhang CL, Bao K, Ma XJ. Prosthesis replacement of the proximal humerus after the resection of bone tumors. Chin J Cancer 2010; 29: 121-4.

\section{Address for correspondence}

\section{Tomasz Goryń}

Department of Soft Tissue/Bone Sarcoma and Melanoma Memorial Cancer Center and Institute of Oncology

W.K. Roentgena 5

02-781 Warsaw, Poland

e-mail: praktyka.goryn@wp.pl

Submitted: 23.05 .2017

Accepted: 12.06 .2017 